\title{
THE DETERMINANT OF THE DIRICHLET-TO-NEUMANN MAP FOR SURFACES WITH BOUNDARY
}

\author{
COLIN GUILLARMOU AND LAURENT GUILLOPÉ
}

\begin{abstract}
For any orientable compact surface with boundary, we compute the regularized determinant of the Dirichlet-to-Neumann (DN) map in terms of particular values of dynamical zeta functions by using natural uniformizations, one due to Mazzeo-Taylor, the other to Osgood-Phillips-Sarnak. We also relate in any dimension the DN map for the Yamabe operator to the scattering operator for a conformally compact related problem by using uniformization.
\end{abstract}

\section{INTRODUCTION}

Let $(\bar{X}, \bar{g})$ be a connected compact Riemannian manifold with boundary, then the Dirichletto-Neumann (DN) map is the map

$$
\mathcal{N}: C^{\infty}(\partial \bar{X}) \rightarrow C^{\infty}(\partial \bar{X})
$$

defined by the following problem: let $f \in C^{\infty}(\partial \bar{X})$ and let $u \in C^{\infty}(\bar{X})$ be the solution of

$$
\Delta_{\bar{g}} u=0,\left.\quad u\right|_{\partial \bar{X}}=f,
$$

then if $\partial_{n}$ is the interior pointing vector field which is normal to $\partial \bar{X}$, we set

$$
\mathcal{N} f:=-\left.\partial_{n} u\right|_{\partial \bar{X}} .
$$

It is well-known that $\mathcal{N}$ is an elliptic self-adjoint pseudo-differential operator on $\partial \bar{X}$ with principal symbol $|\xi|_{h_{0}}, \xi \in T^{*} \partial \bar{X}$, if $h_{0}:=\left.g\right|_{T \partial \bar{X}}$ (see [36, 7.11] for example). It is then possible to define its determinant by the Ray-Singer method [33]. Indeed, if $A$ is an elliptic self-adjoint pseudo-differential operator of order $p>0$ with positive principal symbol, we can set, following [35, 33, 24,

$$
\operatorname{det}(A)=e^{-\partial_{s} \zeta_{A}(0)}, \quad \zeta_{A}(s)=\operatorname{Tr}\left(A^{-s}\right)
$$

where $\zeta_{A}(s)$ is a priori defined for $\Re(s) \gg 0$ but has a meromorphic extension to $\mathbb{C}$ with no pole at $s=0$. If we apply this to $A=\mathcal{N}$, we obtain $\operatorname{det}(\mathcal{N})=0$ since ker $\mathcal{N} \neq 0$, indeed by Green's identity one has $\int_{\partial \bar{X}} \mathcal{N} f \cdot f \operatorname{dvol}_{h_{0}}=\int_{\bar{X}}|\nabla u|^{2} \operatorname{dvol}_{\bar{g}}$ thus ker $\mathcal{N}=\mathbb{R}$ is the space of constant functions on $\partial \bar{X}$. We then have to modify the definition of $\operatorname{det}(\mathcal{N})$ : if $\Pi$ is the orthogonal projection in $L^{2}\left(\partial \bar{X}, \mathrm{~d} v o l_{h_{0}}\right)$ onto the kernel ker $\mathcal{N}$, we take $\operatorname{det}^{\prime} \mathcal{N}$ defined by

$$
\operatorname{det}^{\prime}(\mathcal{N})=e^{-\partial_{s} \zeta_{\mathcal{N}}^{*}(0)}, \quad \zeta_{\mathcal{N}}^{*}(s)=\operatorname{Tr}\left(\mathcal{N}^{-s}(1-\Pi)\right)
$$

which is well defined as before. To compute $\operatorname{det}^{\prime}(\mathcal{N})$, we first show the

Theorem 1.1. If $(\bar{X}, \bar{g})$ is a Riemannian surface with boundary and if $\ell_{\bar{g}}(\partial \bar{X})$ is the length of the boundary $\partial \bar{X}$ for the metric $\bar{g}$, the value $\operatorname{det}^{\prime}(\mathcal{N}) / \ell_{\bar{g}}(\partial \bar{X})$ is a conformal invariant of the conformal manifold with boundary $(\bar{X},[\bar{g}])$.

Note that this was proved in the case where $\bar{X}$ is a topological disc by Edward-Wu [7].

Consequently, it is sufficient to study the case of particular conformal representative in the conformal class, that is to use uniformization.

The first natural uniformization we will use has been proved by Mazzeo and Taylor [26, it picks a complete constant negative curvature metric in the conformal class: indeed, they show that there exists a unique conformally compact metric $g$ on the interior $X$ of $\bar{X}$ such that $g$ has curvature -1 and $g$ is conformal to $\bar{g}$. The manifold $(X, g)$ is then isometric to an infinite volume 
quotient $X \simeq \Gamma \backslash \mathbb{H}^{2}$ of the hyperbolic plane by a convex co-compact group of isometries. We use this uniformization to compute $\operatorname{det}^{\prime}(\mathcal{N})$, although the DN map in this case does not really make sense, but instead we have the scattering operator.

Before stating the result, we need to recall a few definitions about Riemann surfaces and their Selberg (resp. Ruelle) zeta function. Let $\Gamma \subset \operatorname{Isom}^{+}\left(\mathbb{H}^{2}\right)$ be a Fuchsian subgroup with only hyperbolic elements (i.e. fixing 2 points at the boundary of $\mathbb{H}^{2}$ ), the quotient $X=\Gamma \backslash \mathbb{H}^{2}$ is a geometrically finite complete hyperbolic manifold. We recall that any $\gamma \in \Gamma$ is conjugated to the dilation $z \rightarrow e^{\ell(\gamma)} z$, with translation length $\ell(\gamma) \in \mathbb{R}^{+}$in the hyperbolic half-plane model $\mathbb{H}^{2}=\{z \in \mathbb{C} ; \Im(z)>0\}$, note that the set $[\Gamma]$ of primitive conjugacy classes of $\Gamma$ is in one-to-one correspondence with the set $[C]$ of primitive closed oriented geodesics $c$, the length of the closed geodesic $c$ corresponding to $\gamma$ being equal to $\ell(\gamma)$. There is a dynamical Ruelle type zeta function defined by the formula

$$
R_{\Gamma}(\lambda):=\prod_{[\gamma] \in[\Gamma]}\left(1-e^{-\lambda \ell(\gamma)}\right)
$$

and the Selberg zeta function

$$
Z_{\Gamma}(\lambda):=\prod_{k \in \mathbb{N}_{0}} R_{\Gamma}(\lambda+k)
$$

These products converge for $\Re(\lambda)>\delta$ where $\delta \in[0,1]$ is the exponent of convergence of the Poincaré series of $\Gamma$, equal to 1 only if $\Gamma$ is cocompact. Moreover they admit an analytic extension ${ }^{2}$ to $\mathbb{C}$ and verify the identity

$$
R_{\Gamma}(\lambda)=Z_{\Gamma}(\lambda) / Z_{\Gamma}(\lambda+1) .
$$

We are able to compute the determinant of the DN map $\mathcal{N}$ using the uniformization of [26]:

Theorem 1.2. Let $(\bar{X}, \bar{g})$ be a smooth compact orientable connected Riemannian surface of Euler characteristic $\chi(\bar{X})$, with boundary $\partial \bar{X}$ of length $\ell(\partial \bar{X})$, and let $\mathcal{N}$ be the Dirichlet-to-Neumann operator of $\Delta_{\bar{g}}$ on $\partial \bar{X}$. Let $g$ be the unique up to isometry, infinite volume, complete hyperbolic metric $g$ on $X$ conformal to $\bar{g}_{X}$ and let $\Gamma \subset \operatorname{Isom}^{+}\left(\mathbb{H}^{2}\right)$ the geometrically finite Fuchsian group such that $(X, g)$ is isometric to the space form $\Gamma \backslash \mathbb{H}^{2}$. If we denote by $R_{\Gamma}(\lambda)$ the Ruelle zeta function of $\Gamma$, we have

$$
\frac{\operatorname{det}^{\prime}(\mathcal{N})}{\ell(\partial \bar{X})}= \begin{cases}1 & \text { if } \chi(\bar{X})=1 \\ \ell(\gamma) / \pi & \text { if } \chi(\bar{X})=0 \\ {\left[(2 \pi \lambda)^{\chi(\bar{X})-1} R_{\Gamma}(\lambda)\right]_{\mid \lambda=0} / \chi(\bar{X})} & \text { if } \chi(\bar{X})<0\end{cases}
$$

In the second case $\chi(\bar{X})=0$, the group $\Gamma$ is cyclic elementary, generated by the hyperbolic isometry $\gamma$ with translation length $\ell(\gamma)$, length of the unique closed geodesic of the cylinder $X \simeq \Gamma \backslash \mathbb{H}^{2}$.

The proof of this theorem is based on a functional equation for Selberg zeta function for convex co-compact groups obtained in previous work [17] and the observation that the DN map for $(\bar{X}, \bar{g})$ is, modulo constant, the scattering operator $\mathcal{S}(\lambda)$ of the uniformized non-compact manifold $\Gamma \backslash \mathbb{H}^{2}$ at the parameter value $\lambda=1$ : this is discussed in more generality at the end of the introduction. We emphasize that the Theorem holds even when the boundary has more than one connected component, an important fact that we need in the proof being that $\operatorname{ker} \mathcal{N}$ is always equal to the space of constants and not the locally constant functions.

\footnotetext{
${ }^{1}$ The original zeta function of Ruelle was actually defined by the inverse of this one, we prefer to use the convention of Fried 9 .

${ }^{2}$ For the compact case, this is a consequence of Selberg trace formula, here this follows from Fried [9] and Patterson-Perry [31] for instance.
} 
Remark 1: Note that for odd dimensional closed hyperbolic manifolds $X=\Gamma \backslash \mathbb{H}^{d}$, the value $\left|R_{\Gamma}(0)\right|$ for some acyclic representation of the $\pi_{1}$ of the unit tangent bundle $S X$ is the Reidemeister torsion of $S X$ by a result of Fried [10].

Remark 2: It is also worth to say that the proof shows that 0 is always a resonance of multiplicity 1 with resonant state 1 for the Laplacian on any convex co-compact surface except when it's a cylinder where it is then of multiplicity 2 ; as a byproduct it also gives the exact order of vanishing of $R_{\Gamma}(\lambda)$ at $\lambda=0$, which was not apparently known in that case.

The next natural uniformizations for oriented compact surfaces with boundary are given by Osgood-Phillips-Sarnak [29] (see also Brendle [3]), they are of two types: each conformal class of a metric on an oriented compact surface with boundary has a unique

- metric with constant Gauss curvature and with totally geodesic boundary.

- flat metric with constant geodesic curvature boundary.

The Gauss curvature $K$ on $\bar{X}$ and the geodesic curvature $k$ of the boundary $\partial \bar{X}$ are linked through the Gauss-Bonnet formula

$$
\int_{\bar{X}} K \operatorname{dvol}_{g}+\int_{\partial \bar{X}} k \mathrm{~d} \ell_{g}=2 \pi \chi(\bar{X}) .
$$

The flat uniformization has been used by Edward-Wu [7] to show that $\operatorname{det}^{\prime}(\mathcal{N})=\ell(\partial \bar{X})$ for a topological disc (i.e. $\chi(\bar{X})=1$ ), their explicit computation is possible thanks to the circular symmetry of the uniformized flat disc with constant geodesic curvature. In a similar way, we give in the appendix the explicit computation for the planar annulus whose boundary is the union of two concentric circles and show that it fits with the value found in Theorem 1.2 for the hyperbolic cylinder conformal to this annulus. In the case $\chi(\bar{X})<0$, the computation of $\operatorname{det}^{\prime}(\mathcal{N})$ in terms of geometric quantities by using the flat uniformization does not seem apparent at all. As for the constant curvature with geodesic boundary uniformization, the topological disc (i.e. $\chi(\bar{X})>0)$ is uniformized by a half-sphere of curvature +1 , the topological cylinder (i.e. $\chi(\bar{X})=0)$ by a flat cylinder $[0, L] \times S^{1}$ and in both cases, the value $\operatorname{det}^{\prime}(\mathcal{N})$ can be easily computed using decomposition in spherical harmonics of the Laplacian, essentially like for the flat uniformization. However, when $\chi(\bar{X})<0$, the constant curvature -1 uniformization with totally geodesic boundary appears to be more useful to compute $\operatorname{det}^{\prime}(\mathcal{N})$. Indeed it yields a metric on $\bar{X}$ which is isometric to $G_{0} \backslash \mathbb{H}^{2}$ for some discrete group $G_{0}$ of isometries of $\mathbb{H}^{2}$ (containing symmetries of order 2) and the double of $G_{0} \backslash \mathbb{H}^{2}$ along the boundary is the closed hyperbolic surface $M:=G \backslash \mathbb{H}^{2}$ where $G:=G_{0} \cap \operatorname{Isom}^{+}\left(\mathbb{H}^{2}\right)$ is the index 2 subgroup of orientation preserving isometries of $G_{0}$. The Mayer-Vietoris formula for determinants by Burghelea-Friedlander-Kappeler [4 reads in this case

$$
\frac{\operatorname{det}^{\prime}(\mathcal{N})}{\ell(\partial \bar{X})}=-\frac{1}{2 \pi \chi(\bar{X})} \frac{\operatorname{det}^{\prime}\left(\Delta_{G \backslash \mathbb{H}^{2}}\right)}{\left(\operatorname{det}\left(\Delta_{G_{0} \backslash \mathbb{H}^{2}}\right)\right)^{2}}
$$

where $\Delta_{G_{0} \backslash \mathbb{H}^{2}}$ is the Dirichlet realization of the Laplacian on $G_{0} \backslash \mathbb{H}^{2}$. We are thus interested in the value of the regularized determinants of these Laplacians. The determinant $\operatorname{det}^{\prime}\left(\Delta_{G \backslash \mathbb{H}^{2}}\right)$ has been computed by Sarnak and Voros 34, 37. in terms of the derivative at $\lambda=1$ of the Selberg zeta function $Z_{G}(\lambda)$ defined by (1.2) with $\Gamma=G$. Using a trace formula of [19], we prove a similar formula for $\operatorname{det}\left(\Delta_{G_{0} \backslash \mathbb{H}^{2}}\right)$ in terms of a Selberg zeta function $Z_{G_{0}}(\lambda)$ at $\lambda=1$, where the natural Ruelle and Selberg zeta functions $R_{G_{0}}(\lambda), Z_{G_{0}}(\lambda)$ for this case with boundary are defined as follows (see [19, Section 5]): let $\ell_{1}, \ldots, \ell_{N}$ be the lengths of the geodesic boundary components of $\bar{X}$ and let $[C]$ be the set of primitive oriented closed geodesics $c$ of length $\ell_{c}$ and with $n_{c}$ geometric reflections (according to the geometric optic law) on $\partial \bar{X}$, then the zeta 
function $\sqrt{3}$ are defined by the following products:

$$
\begin{gathered}
R_{\partial \bar{X}}(\lambda):=\prod_{j=1}^{N}\left(1-e^{-\lambda \ell_{j}}\right)^{2}, \quad R_{G_{0}}(\lambda):=\prod_{c \in[C]}\left(1-(-1)^{n_{c}} e^{-\lambda \ell_{c}}\right)\left(1-e^{-(\lambda+1) \ell_{c}}\right), \\
Z_{G_{0}}(\lambda):=\prod_{k \in \mathbb{N}_{0}} R_{\partial \bar{X}}(\lambda+2 k) R_{G_{0}}(\lambda+2 k) .
\end{gathered}
$$

We show the

Theorem 1.3. Let $(\bar{X}, \bar{g})$ be a compact oriented surface with boundary, with negative Euler characteristic $\chi(\bar{X})$. Let $\bar{g}_{0}$ be the unique, up to isometry, constant negative curvature metric with totally geodesic boundary on $\bar{X}$ and let $G_{0} \subset \operatorname{Isom}\left(\mathbb{H}^{2}\right)$ be the discrete group such that $\left(\bar{X}, \bar{g}_{0}\right)$ is isometric to $G_{0} \backslash \mathbb{H}^{2}$. Let $G=G_{0} \cap \mathrm{Isom}^{+}\left(\mathbb{H}^{2}\right)$ be the subgroup of $G_{0}$ of orientation preserving isometries and $Z_{G_{0}}(\lambda), Z_{G}(\lambda)$ be the associated Selberg zeta function of $G_{0}$ and $G$, then

$$
\frac{\operatorname{det}^{\prime}(\mathcal{N})}{\ell(\partial \bar{X})}=-\frac{Z_{G}^{\prime}(1)}{\left(Z_{G_{0}}(1)\right)^{2}} \frac{e^{\ell(\partial \bar{X}) / 4}}{2 \pi \chi(\bar{X})}
$$

Thus, if $\Gamma \backslash \mathbb{H}^{2}$ is the uniformization of $(\bar{X}, \bar{g})$ given by Theorem 1.2, then

$$
\left[\lambda^{\chi(\bar{X})-1} R_{\Gamma}(\lambda)\right]_{\mid \lambda=0}=-\frac{Z_{G}^{\prime}(1)}{Z_{G_{0}}(1)^{2}} e^{\frac{\ell(\partial \bar{X})}{4}}(2 \pi)^{-\chi(\bar{X})} .
$$

Although the products defining $R_{\Gamma}(\lambda), Z_{G}(\lambda), Z_{G_{0}}(\lambda)$ do not converge, we can view the last identity of Theorem 1.3 as a relation between length spectrum of $\Gamma \backslash \mathbb{H}^{2}$ and $G_{0} \backslash \mathbb{H}^{2}$, which does not appear obvious at all. Let us also remark that the determinant of the Laplacian on an hyperbolic compact surface has different expressions with Selberg zeta values, the Sarnak-Voros [34, 37] one related to the Fuchsian uniformization and the McIntyre-Takhtajan [27] related to the Schottky uniformization.

In the last section we discuss in more generality (in higher dimension) the relation between Dirichlet-to-Neumann map and scattering operator. An $(n+1)$-dimensional asymptotically hyperbolic manifold $(X, g)$ is a complete Riemannian non-compact manifold, which is the interior of a smooth compact manifold with boundary $\bar{X}$ such that for any boundary defining function $x$ of $\partial \bar{X}$ (i.e. $\partial \bar{X}=\{x=0\}$ and $\left.d x\right|_{\partial \bar{X}} \neq 0$ ), then $\bar{g}=x^{2} g$ is a smooth metric on $\bar{X}$ such that $|d x|_{x^{2} g}=1$ on $\partial \bar{X}$. The metric $h_{0}:=\left.\bar{g}\right|_{T \partial \bar{X}}$ induced on $\partial \bar{X}$ depends on $x$ and another choice of $x$ yields a metric on $\partial \bar{X}$ conformal to $h_{0}$, we thus define the conformal infinity of $(X, g)$ as the conformal class of $\left[h_{0}\right]$ on $\partial \bar{X}$. There is a natural meromorphic family of operators (defined in Section 2) $\mathcal{S}(\lambda)$ (for $\lambda \in \mathbb{C}$ ) called scattering operator, acting on $C^{\infty}(\partial \bar{X})$, these are elliptic conformally covariant pseudo-differential operators of order $2 \lambda-n$ with principal symbol $|\xi|_{h_{0}}^{2 \lambda-n}$ where $h_{0}=\left.\bar{g}\right|_{T \partial \bar{X}}$ is a conformal representative of the conformal infinity of $(X, g)$. When $g$ is Einstein, Graham and Zworski [15] showed that $\mathcal{S}(n / 2+k)$ for $k \in \mathbb{N}$ are conformal powers of the Laplacian on the boundary $\partial \bar{X}$, initially defined in [13]. Since $\mathcal{S}(\lambda)$ has order 1 when $\lambda=(n+1) / 2$ and the same principal symbol than a Dirichlet-to-Neumann map on the compact manifold $(\bar{X}, \bar{g})$, we may expect that it is realized as a DN map for an elliptic compact problem with boundary. We observe that when $g$ has constant scalar curvature (for instance if $g$ is Einstein), then $\mathcal{S}\left(\frac{n+1}{2}\right)$ is the Dirichlet-to-Neumann map of the conformal Laplacian on a whole class of smooth metric $\bar{g}$ on $\bar{X}$, conformal to $g$, with $\left.\bar{g}\right|_{T \partial \bar{X}}=h_{0}$ and with minimal boundary $\partial \bar{X}$. Conversely it is clear that there is no constant curvature uniformization when $n+1>2$, but instead there is a solution of a singular Yamabe problem, that is, for a given $(\bar{X}, \bar{g})$, there exists an asymptotically hyperbolic metric with constant scalar curvature on the interior $X$ in the conformal class of $\bar{g}$. The existence and regularity of such a solution of this singular Yamabe problem is due to Aviles-Mac Owen [2, Mazzeo [25] and Andersson-ChruścielFriedrich [1]. If $K$ is the mean curvature of $\partial \bar{X}$ for $\bar{g}$ and $\mathcal{N}$ is the DN map for the conformal

\footnotetext{
${ }^{3}$ The function we use is actually the square root of that of [19].
} 
Laplacian $P=\Delta_{\bar{g}}+\operatorname{Scal}_{\bar{g}}(n-1) /(4 n)$, we show that $\mathcal{N}+(n-1) K / 2$ is the value $\mathcal{S}((n+1) / 2)$ for a complete manifold with constant negative scalar curvature, conformal to $\bar{g}$ on the interior $X$ of $\bar{X}$. Note that $\mathcal{N}+(n-1) K / 2$ is known to be the natural conformally covariant operator on the boundary associated to $P$, see [5].

Acknowledgement. We thank E. Aubry, P. Delanoë, M. Harmer, A. Hassell, R. Mazzeo and P. Perry for useful discussions and for pointing out the right references. C.G. acknowledges support of NSF grant DMS0500788, and french ANR grants JC05-52556 and JC0546063.

\section{Computation of $\operatorname{det}^{\prime}(\mathcal{N})$ using Mazzeo-Taylor uniformization}

We now recall the definition of the scattering operator $\mathcal{S}(\lambda)$ on an asymptotically hyperbolic manifold $(X, g)$ of dimension $n+1$. From Graham-Lee [14, for any choice $h_{0}$ in the conformal infinity $\left[h_{0}\right]$, such a metric can be written uniquely in a collar neighbourhood $[0, \epsilon)_{x} \times \partial \bar{X}$ of the boundary under the form

$$
g=\frac{d x^{2}+h(x)}{x^{2}}, \quad h(0)=h_{0}
$$

for some smooth 1-parameter family of metric $h(x)$ on $\partial \bar{X}$ ( $x$ is a boundary defining function of $\partial \bar{X})$. If $h(x)$ has a Taylor expansion at $x=0$ with only even powers of $x$, then $g$ is called even (see [16]). If $g$ is even and $f \in C^{\infty}(\partial \bar{X}), \Re(\lambda) \geq n / 2$ and $\lambda \notin n / 2+\mathbb{N}$, then $\mathcal{S}(\lambda) f:=$ $\left.c(\lambda) u_{\lambda}^{+}\right|_{\partial \bar{X}} \in C^{\infty}(\partial \bar{X})$ wherब $4(\lambda)$ is the normalisation constant $c(\lambda):=2^{2 \lambda-n} \Gamma\left(\lambda-\frac{n}{2}\right) / \Gamma\left(\frac{n}{2}-\lambda\right)$ and $u_{\lambda}, u_{\lambda}^{ \pm}$are defined by solving the Poisson problem [15]

$$
\left(\Delta_{g}-\lambda(n-\lambda)\right) u_{\lambda}=0, \quad u_{\lambda}=x^{n-\lambda} u_{\lambda}^{-}+x^{\lambda} u_{\lambda}^{+}, \quad u_{\lambda}^{ \pm} \in C^{\infty}(\bar{X}),\left.\quad u_{\lambda}^{-}\right|_{\partial \bar{X}}=f .
$$

We see that $\mathcal{S}(\lambda) f$ depends on $g$ and on the choice of $x$ or equivalently on the choice of conformal representative $h_{0}=\left.x^{2} g\right|_{T \partial \bar{X}}$ of the conformal infinity of $(X, g)$. Changing $h_{0}$ into $\hat{h}_{0}=e^{2 \omega_{0}} h_{0}$ with $\omega_{0} \in C^{\infty}(\partial \bar{X})$ induces the scattering operator

$$
\hat{\mathcal{S}}(\lambda)=e^{-\lambda \omega_{0}} \mathcal{S}(\lambda) e^{(n-\lambda) \omega_{0}} .
$$

From [22, 15], $\mathcal{S}(\lambda)$ is holomorphic in the half plane $\{\lambda \in \mathbb{C} ; \Re(\lambda)>n / 2\}$, moreover it is a pseudodifferential operator of order $2 \lambda-n$ with principal symbol $|\xi|_{h_{0}}^{2 \lambda-n}$ (thus elliptic) and it is self-adjoint when $\lambda \in(n / 2,+\infty)$, which makes its zeta regularized determinant well defined by 24]. If the dimension $n+1$ is even, one shows easily that if $\hat{h}_{0}$ is conformal to $h_{0}$, the conformal relation (2.3) between the associated operators $\mathcal{S}(\lambda)$ and $\hat{\mathcal{S}}(\lambda)$ implies that $\operatorname{det}(\mathcal{S}(\lambda))=\operatorname{det}(\hat{\mathcal{S}}(\lambda))$, see [17, Sec. 4] for instance.

We are back to our case of surfaces (here $n=1)$, thus let $(\bar{X}, \bar{g})$ be a smooth Riemannian surface with boundary. We first relate $\mathcal{N}$ to the scattering operator of an associated non-compact hyperbolic surface. Let $\rho$ be a function that defines $\partial \bar{X}$ and such that $\bar{g}=d \rho^{2}+h_{0}+O(\rho)$ for some metric $h_{0}$ on $\partial \bar{X}$, so the normal vector field to the boundary is $\partial_{n}=\partial_{\rho}$ on $\partial \bar{X}$. Let $g=\hat{\rho}^{-2} \bar{g}$ be the unique complete hyperbolic metric on the interior $X$ of $\bar{X}$, obtained by Mazzeo-Taylor [26], where $\hat{\rho}=\rho+O\left(\rho^{2}\right)$ is some smooth function on $\bar{X}$, then $(X, g)$ is an asymptotically hyperbolic manifold in the sense stated in the introduction. Then $g$ is even since the metric outside some compact is the metric on a hyperbolic funnel, that is $d r^{2}+\cosh ^{2}(r) d t^{2}$ on $(0, \infty)_{r} \times(\mathbb{R} / a \mathbb{Z})_{t}$ for some $a>0$ (it suffices to set $x=e^{-r}$ to have a model form (2.1)). Therefore the geodesic function $x$ such that $g$ is like (2.1) implies $h(x)=h_{0}+O\left(x^{2}\right)$ and $x=\rho+O\left(\rho^{2}\right)$. By studying the Poisson problem at energy $\lambda$ close to 1 for $\Delta_{g}$, for any $f \in C^{\infty}(\partial \bar{X})$, there exists a unique $u_{\lambda} \in C^{\infty}(X)$ such that

$$
\left(\Delta_{g}-\lambda(1-\lambda)\right) u_{\lambda}=0, \quad u_{\lambda} \sim_{x \rightarrow 0} x^{1-\lambda}\left(f+\sum_{j=1}^{\infty} x^{2 j} f_{2 j}^{-}(\lambda)\right)+x^{\lambda}\left(c(\lambda) \mathcal{S}(\lambda) f+\sum_{j=1}^{\infty} x^{2 j} f_{2 j}^{+}(\lambda)\right)
$$

\footnotetext{
${ }^{4}$ We changed the convention since in the literature, $\left.u_{\lambda}^{+}\right|_{\partial \bar{X}}$ would be the scattering operator acting on $f$.
} 
for some $f_{2 j}^{ \pm}(\lambda) \in C^{\infty}(\partial \bar{X})$ (we used evenness of the metric so that odd powers of $x$ are zeros, see [15]). In particular at $\lambda=1$ we have $u:=u_{1} \in C^{\infty}(\bar{X})$ and $\Delta_{g} u=0$ but $\Delta_{g}=\hat{\rho}^{2} \Delta_{\bar{g}}$ thus

$$
\Delta_{\bar{g}} u=0, \quad u \in C^{\infty}(\bar{X}), \quad u=f-x \mathcal{S}(1) f+O\left(\rho^{2}\right),
$$

but since $\partial_{x}=\partial_{\hat{\rho}}=\partial_{\rho}=\partial_{n}$ on $\partial \bar{X}$ we automatically get

Lemma 2.1. The Dirichlet-to-Neumann map $\mathcal{N}$ for $\Delta_{\bar{g}}$ is given by the scattering operator $\mathcal{S}(1)$ at energy 1 for the Laplacian $\Delta_{g}$ on the asymptotically hyperbolic surface $(X, g)$ conformal to $\bar{g}$, where $\mathcal{S}(\lambda)$ is defined using the boundary defining function associated to the representative $h_{0}=\left.\bar{g}\right|_{T \partial \bar{X}}$ of the conformal infinity $\left[h_{0}\right]$ of $(X, g)$.

Taking a conformal metric $\bar{g}_{1}=e^{2 \omega} \bar{g}$ on $\bar{X}$ gives a Laplacian $\Delta_{\bar{g}_{1}}=e^{-2 \omega} \Delta_{\bar{g}}$ and the normal vector field to the boundary becomes $\partial_{n}=e^{-\omega_{0}} \partial_{x}$ where $\omega_{0}=\left.\omega\right|_{\partial \bar{X}}$. We deduce that the associated Dirichlet-to-Neumann map $\mathcal{N}_{1}$ satisfies $\mathcal{N}_{1}=e^{-\omega_{0}} \mathcal{N}$.

Theorem 2.2. Let $\bar{g}_{0}$ and $\bar{g}_{1}=e^{2 \omega} \bar{g}_{0}$ be two conformally related metrics on a surface with boundary $\bar{X}$, and let $\mathcal{N}_{0}, \mathcal{N}_{1}$ be the respective Dirichlet-to-Neumann operators. Then $\operatorname{det}^{\prime}\left(\mathcal{N}_{0}\right) / \ell_{\bar{g}_{0}}(\partial \bar{X})=$ $\operatorname{det}^{\prime}\left(\mathcal{N}_{1}\right) / \ell_{\bar{g}_{1}}(\partial \bar{X})$ where $\ell_{\bar{g}_{i}}(\partial \bar{X})$ is the length of the boundary for the metric $\bar{g}_{i}, i=0,1$.

Proof: By the main formula of Paycha-Scott [32] (see also [28]),

$$
\operatorname{det}^{\prime}\left(\mathcal{N}_{i}\right)=\exp \left(\operatorname{TR}\left(\log \left(\mathcal{N}_{i}\right)\left(1-\Pi_{i}\right)\right)\right), \quad i=0,1
$$

where TR is the Kontsevich-Vishik canonical trace defined in [24], $\log \left(\mathcal{N}_{i}\right)$ is defined by a contour integral (see 24, 32 for details), and $\Pi_{i}$ the orthogonal projection onto ker $\mathcal{N}_{i}$ with respect to the volume density on $\partial \bar{X}$ induced by $h_{i}:=\left.\bar{g}_{i}\right|_{\partial \bar{X}}$, i.e. the projection onto the constants for the volume density $\mathrm{dvol}_{h_{i}}$. It is important to note that this formula holds (i.e. Guillemin-Wodzicki residue trace does not show-up in the formula) since the DN maps $\mathcal{N}_{i}$ have regular parity in the sense of [17, Sect. 2] and thus $\log \mathcal{N}_{i}$ as well: indeed, take the Mazzeo-Taylor uniformization $g$ of $\bar{g}_{0}$ (which is the same than that of $\bar{g}_{1}$ ), then Proposition 3.6 of [17] shows that the scattering operator $\mathcal{S}(\lambda)$ associated to $g$ has regular parity in the sense of [17, Sect. 2] since the hyperbolic metric $g$ is even; this implies by using Lemma 2.1 that $\mathcal{N}_{i}$ has regular parity for $i=1,2$. If $g_{t}=e^{2 t \omega} g_{0}$ is a conformal change and $h_{t}=\left.g_{t}\right|_{\partial \bar{X}}=e^{2 t \omega_{0}} h_{0}$ where $\omega_{0}=\left.\omega\right|_{\partial \bar{X}}$, then the DN map for the metric $g_{t}$ is unitarily equivalent to the self-adjoint operator $\mathcal{N}_{t}:=e^{-t \frac{\omega}{2}} \mathcal{N}_{0} e^{-t \frac{\omega}{2}}$ on $L^{2}\left(\partial \bar{X}, \operatorname{dvol}_{h_{0}}\right)$, with $L^{2}$ kernel projector

$$
\Pi_{t}=\left(\ell_{h_{t}}(\partial \bar{X})\right)^{-1} e^{t \frac{\omega}{2}} \otimes e^{t \frac{\omega}{2}}
$$

where $\ell_{h_{t}}(\partial \bar{X})$ is the length of $\partial \bar{X}$ for the metric $h_{t}$. First we have that

$$
\partial_{t}\left(\log \left(\mathcal{N}_{t}\right)\left(1-\Pi_{t}\right)\right)=\left(\partial_{t} \mathcal{N}_{t}\right) \mathcal{N}_{t}^{-1}\left(1-\Pi_{t}\right)-\log \left(\mathcal{N}_{t}\right) \partial_{t} \Pi_{t}
$$

where $\mathcal{N}_{t}^{-1}$ is the unique operator (modulo $\Pi_{t}$ ) satisfying $\mathcal{N}_{t} \mathcal{N}_{t}^{-1}=\mathcal{N}_{t}^{-1} \mathcal{N}_{t}=1-\Pi_{t}$, that is $\mathcal{N}_{t}^{-1}=\left(1-\Pi_{t}\right) e^{t \frac{\omega_{0}}{2}} \mathcal{N}_{0}^{-1} e^{t \frac{\omega_{0}}{2}}\left(1-\Pi_{t}\right)$ : indeed, multiplying on the left by $\mathcal{N}_{t}$ gives

$$
\begin{aligned}
\mathcal{N}_{t}\left(1-\Pi_{t}\right) e^{t \frac{\omega_{0}}{2}} \mathcal{N}_{0}^{-1} e^{t \frac{\omega_{0}}{2}}\left(1-\Pi_{t}\right) & =e^{-t \frac{\omega_{0}}{2}}\left(1-\Pi_{0}\right) e^{t \frac{\omega_{0}}{2}}\left(1-\Pi_{t}\right) \\
& =\left(1-\ell_{h_{t}}(\partial \bar{X}) e^{-t \omega_{0}} \Pi_{t}\right)\left(1-\Pi_{t}\right)=1-\Pi_{t}
\end{aligned}
$$

and the same holds by multiplying on the right by $\mathcal{N}_{t}$. Thus taking the log derivative of $\operatorname{det}^{\prime}\left(\mathcal{N}_{t}\right)$ with respect to $t$ gives (by the same arguments than [17, Sec. 4]) that

$$
\partial_{t} \log \left(\operatorname{det}^{\prime}\left(\mathcal{N}_{t}\right)\right)=-\operatorname{TR}\left(\omega_{0}\left(1-\Pi_{t}\right)\right)-\operatorname{Tr}\left(\left(\log \left(\mathcal{N}_{t}\right) \partial_{t} \Pi_{t}\right)\right.
$$

where $\mathrm{Tr}$ is the usual trace. Using (2.6) , we compute $\partial_{t} \Pi_{t}=\frac{1}{2}\left(\omega_{0} \Pi_{t}+\Pi_{t} \omega_{0}\right)+\partial_{t}\left(\ell_{h_{t}}(\partial \bar{X})^{-1}\right) \Pi_{t}$ but since $\log \left(\mathcal{N}_{t}\right) \Pi_{t}=\Pi_{t} \log \left(\mathcal{N}_{t}\right)=0$ and the trace is cyclic, we have

$$
\operatorname{Tr}\left(\left(\log \left(\mathcal{N}_{t}\right) \partial_{t} \Pi_{t}\right)=\operatorname{Tr}\left(\log \left(\mathcal{N}_{t}\right) \omega_{0} \Pi_{t}\right)=\operatorname{Tr}\left(\Pi_{t} \log \left(\mathcal{N}_{t}\right)\right)=0\right.
$$


Now TR $\left(\omega_{0}\right)=0$ since the Kontsevich-Vishik trace of a differential operator is 0 in odd dimension (see [24]), but the trace of a smoothing operator is the integral on the diagonal of its Schwartz kernel, therefore

$$
\operatorname{TR}\left(\omega_{0} \Pi_{t}\right)=\operatorname{Tr}\left(\omega_{0} \Pi_{t}\right)=\frac{\int_{\partial \bar{X}} \omega_{0} e^{t \omega_{0}} \operatorname{dvol}_{h_{0}}}{\int_{\partial \bar{X}} e^{t \omega_{0}} \operatorname{dvol}_{h_{0}}}=\partial_{t} \log \left(\ell_{h_{t}}(\partial \bar{X})\right)
$$

Then integrating in $t \in[0,1]$ we get the right law for the determinant

We now prove Theorem 1.2 .

Proof of Theorem 1.2. Since $\mathcal{S}(1)=\mathcal{N}$, we have to compute $\operatorname{det}^{\prime}(\mathcal{S}(1))$. It is clear that the kernel of $\mathcal{S}(1)$ is one dimensional, composed of the constants, since it is the case for $\mathcal{N}$. According to the main formula of Paycha-Scott 32 we have for $\lambda>1 / 2$

$$
\operatorname{det}(\mathcal{S}(\lambda))=\exp (\operatorname{TR}(\log \mathcal{S}(\lambda))), \quad \operatorname{det}^{\prime}(\mathcal{S}(1))=\exp (\operatorname{TR}(\log (\mathcal{S}(1))(1-\Pi)))
$$

where $\Pi$ is the projection onto the constants. To compute $\operatorname{det}^{\prime}(\mathcal{S}(1))$, we shall analyze $\operatorname{det} \mathcal{S}(\lambda)$ in the neighborhood of $\lambda=1$.

From [17, Th. 1.3] and the fact that $\Delta_{g}$ has no $L^{2}$ zero-eigenvalue, $\operatorname{det}(\mathcal{S}(\lambda))$ has no pole in a neighbourhood of $\lambda=1$ and is holomorphic near $\lambda=1$ with a zero of order $\nu_{1}$ where the multiplicity $\nu_{\lambda_{0}}$ for $\lambda_{0} \in \mathbb{C}$ is defined by

$$
\nu_{\lambda_{0}}:=-\operatorname{Tr}\left(\operatorname{Res}_{\lambda=\lambda_{0}}\left(\partial_{\lambda} \mathcal{S}(\lambda) \mathcal{S}^{-1}(\lambda)\right)\right)
$$

Let us now compute $\nu_{1}$. We consider the largest integer $k$ such that there exists a holomorphic (in $\lambda$ ) family of functions $u_{\lambda}$ in $L^{2}(\partial \bar{X})$ with $u_{1} \in \operatorname{ker} \mathcal{S}(1)$, and such that $\mathcal{S}(\lambda) u_{\lambda}=O\left((\lambda-1)^{k}\right)$. This maximum is achieved for some $u_{\lambda}$, is positive and is exactly $\nu_{1}$ by Gohberg-Sigal theory (see [11] or [18). Thus there exists a family of functions $u_{\lambda}$ on $\partial \bar{X}$, holomorphic in $\lambda$, with $u_{1} \in \operatorname{ker} \mathcal{S}(1)$ such that $\mathcal{S}(\lambda) u_{\lambda}=(\lambda-1)^{\nu_{1}} \psi+O\left((\lambda-1)^{\nu_{1}+1}\right)$ for some function $\psi \neq 0$. Then setting $u_{\lambda}=u_{1}+(\lambda-1) v+O\left((\lambda-1)^{2}\right)$ we get the equation

$$
\mathcal{S}(\lambda) u_{\lambda}=(\lambda-1)\left(\mathcal{S}(1) v+\mathcal{S}^{\prime}(1) u_{1}\right)+O\left((\lambda-1)^{2}\right)
$$

which we multiply with $u_{1}$, integrate and use self adjointness of $\mathcal{S}(1)$ with $\mathbb{R} u_{1}=\operatorname{ker} \mathcal{S}(1)$ to deduce

$$
\left\langle\mathcal{S}(\lambda) u_{\lambda}, u_{1}\right\rangle=(\lambda-1) \int_{\partial \bar{X}} u_{1} \mathcal{S}^{\prime}(1) u_{1} \mathrm{dvol}_{h_{0}}+O\left((\lambda-1)^{2}\right) .
$$

Recall that $u_{1}$ is constant since in the kernel of $\mathcal{N}$, but following the notation of FeffermanGraham [8, Th. 4.3], we have a kind of $Q$ curvature defined by $Q:=-\mathcal{S}^{\prime}(1) 1$ and they prove the identity 6

$$
\int_{\partial \bar{X}} Q \operatorname{dvol}_{h_{0}}=-0-\operatorname{vol}(X)
$$

where $0-\operatorname{vol}(X)$ is the renormalized volume (also called 0 -volume) of $X$, i.e. the constant $V$ in the expansion

$$
\operatorname{Vol}(x>\epsilon)=c_{0} \epsilon^{-1}+V+O(\epsilon), \quad \text { as } \epsilon \rightarrow 0 .
$$

It is however proved, from Gauss-Bonnet formula, by Guillopé-Zworski [21] and Epstein [31, Appendix] that

$$
0-\operatorname{vol}(X)=-2 \pi \chi(\bar{X})=2 \pi(2 g+N-2)
$$

where $\chi(\bar{X})$ is the Euler characteristic of $\bar{X}, g$ is its genus and $N$ the number of boundary components. It follows that the coefficient of $(\lambda-1)$ in (2.8) does not vanish if $\chi(\bar{X}) \neq 0$ and then $\nu_{1}=1$.

\footnotetext{
${ }^{5}$ They actually define $Q:=S^{\prime}(1) 1$ where $S(\lambda):=c(\lambda) \mathcal{S}(\lambda)$, so clearly $Q=-\mathcal{S}^{\prime}(1) 1$ since $\mathcal{S}(1) 1=0$ and $c(1)=-1$.

${ }^{6}$ We emphasize that their proof is only based on Green's identity and evenness of the metric expansion at the boundary. In particular it includes the case of hyperbolic surfaces.
} 
Recall (see [15]) that $\mathcal{S}(\lambda)$ is self-adjoint for $\lambda$ real, Fredholm, analytic in $\lambda$ near $\lambda=1$ and invertible in a small pointed disc (of radius $\epsilon>0$ ) centered at 1 , moreover $\mathcal{S}(1)$ has 0 as isolated eigenvalue of multiplicity 1, then one can use Kato perturbation theory [23, VII,3] to deduce that for $\lambda$ near $\lambda=1$, the spectrum of the operator $\mathcal{S}(\lambda)$ near 0 is an isolated eigenvalue of multiplicity 1 , that we denote $\alpha(\lambda)$; moreover it is holomorphic in $\lambda$ near 1 and there is a holomorphic $L^{2}$ normalized associated eigenvector $w_{\lambda}$. We have $w_{\lambda}=w_{1}+O(\lambda-1)$ where $w_{1}=\ell(\partial \bar{X})^{-1 / 2} \in \operatorname{ker} \mathcal{S}(1), \ell(\partial \bar{X})$ being the length of the curve $\partial \bar{X}$ for the metric $h_{0}$, and we get the equation

$\mathcal{S}(\lambda) w_{\lambda}=\alpha(\lambda) w_{\lambda}, \quad w_{\lambda}=\ell(\partial \bar{X})^{-1 / 2}+(\lambda-1) v+O\left((\lambda-1)^{2}\right), \quad \alpha(\lambda)=(\lambda-1) \beta+O\left((\lambda-1)^{2}\right)$

for some $v \in C^{\infty}(\partial \bar{X})$ and $\beta \in \mathbb{R}$. Taking a Taylor expansion of (2.10) yields

$$
\mathcal{S}(1) v+\mathcal{S}^{\prime}(1) w_{1}=\beta w_{1}
$$

where we used the notation ' for $\partial_{\lambda}$. Pairing as before with $w_{1}$ and using that $\mathcal{S}(1)$ is self adjoint and previous arguments with $\int_{\partial \bar{X}} Q \operatorname{dvol}_{h_{0}}=2 \pi \chi(\bar{X})$ gives

$$
\beta=-\frac{2 \pi \chi(\bar{X})}{\ell(\partial \bar{X})}
$$

Now let $\Pi_{\lambda}$ be the orthogonal projection onto $\operatorname{ker}(\mathcal{S}(\lambda)-\alpha(\lambda))$, and we define the function

$$
h(\lambda):=\exp \left(\operatorname{TR}\left(\log (\mathcal{S}(\lambda))\left(1-\Pi_{\lambda}\right)\right)\right) .
$$

It is analytic near $\lambda=1$ and $\Pi_{1}=\Pi$ thus the limit of $h(\lambda)$ at $\lambda=1$ is $h(1)=\operatorname{det}^{\prime}(\mathcal{S}(1))$ by (2.5), the value we search to compute. For $\lambda \in \mathbb{R}$ close to 1 but $\lambda \neq 1$, we have $\log (\mathcal{S}(\lambda)) \Pi_{\lambda}=$ $\log (\alpha(\lambda)) \Pi_{\lambda}$, thus using the first identity in (2.5) and the fact that TR is the usual trace on finite rank operators, we obtain

$$
h(\lambda)=\exp (\operatorname{TR}(\log \mathcal{S}(\lambda)) \exp (-\log \alpha(\lambda))=\operatorname{det}(\mathcal{S}(\lambda)) / \alpha(\lambda) .
$$

But $\alpha(\lambda)=(\lambda-1) \beta(1+O(\lambda-1))$ by (2.10), which proves that

$$
\operatorname{det}^{\prime}(\mathcal{S}(1))=\lim _{\lambda \rightarrow 1} \frac{\operatorname{det} \mathcal{S}(\lambda)}{\beta(\lambda-1)} .
$$

In [17, we proved the functional equation

$$
\operatorname{det}(\mathcal{S}(\lambda))=\frac{Z_{\Gamma}(1-\lambda)}{Z_{\Gamma}(\lambda)} \exp \left(-2 \pi \chi(\bar{X}) \int_{0}^{\lambda-\frac{1}{2}} t \tan (\pi t) d t\right) .
$$

which, following Voros [37, Eq 7.24, 7.25], can be written under the form

$$
\operatorname{det}(\mathcal{S}(\lambda))=\frac{Z_{\Gamma}(1-\lambda)}{Z_{\Gamma}(\lambda)}\left(\frac{(2 \pi)^{1-2 \lambda} \Gamma(\lambda) G(\lambda)^{2}}{\Gamma(1-\lambda) G(1-\lambda)^{2}}\right)^{-\chi(\bar{X})}
$$

where $G$ is the Barnes function (see [37, Appendix]) which satisfies in particular $\Gamma(z) G(z)=$ $G(z+1)$ and $G(1)=1$. Writing $Z_{\Gamma}(1-\lambda)=R_{\Gamma}(1-\lambda) Z_{\Gamma}(2-\lambda)$ in (2.12) and using that $Z_{\Gamma}(\lambda)$ is holomorphic at $\lambda=1$ implies that

$$
\begin{aligned}
\operatorname{det}^{\prime}(\mathcal{S}(1)) & =-\beta^{-1}\left[\lim _{\lambda \rightarrow 1} \frac{R_{\Gamma}(1-\lambda)}{(1-\lambda)^{1-\chi(\bar{X})}}\right] \lim _{\lambda \rightarrow 1}\left[\frac{(1-\lambda)(2 \pi)^{1-2 \lambda} \Gamma(1-\lambda) \Gamma(\lambda) G(\lambda)^{2}}{G(2-\lambda)^{2}}\right]^{-\chi(\bar{X})} \\
& =(2 \pi)^{\chi(\bar{X})-1} \frac{\ell(\partial \bar{X})}{\chi(\bar{X})} \lim _{\lambda \rightarrow 1} \frac{R_{\Gamma}(1-\lambda)}{(1-\lambda)^{1-\chi(\bar{X})}} .
\end{aligned}
$$

and we are done when $\chi(\bar{X})<0$.

If $\chi(\bar{X})=1, \bar{X}$ is a topological disc and the uniformization that puts a complete hyperbolic metric on $X$ is the usual hyperbolic disc. The proof [17] of the formula (2.12) remains true by 
setting $Z_{\Gamma}(\lambda):=1$ and we can proceed as before where now $\Pi_{\lambda}$ is the projection on the constants $\Pi_{\lambda}=w_{1}\left\langle w_{1},.\right\rangle$ if $w_{1}$ is like above. We finally obtain

$$
\operatorname{det}^{\prime}(\mathcal{S}(1))=-\frac{\ell(\partial \bar{X})}{2 \pi} \lim _{\lambda \rightarrow 1} \frac{\operatorname{det}(\mathcal{S}(\lambda))}{\lambda-1}=\ell(\partial \bar{X})
$$

and we are done for this case. Notice that it matches with the result of Edward-Wu 7 .

The last case $\chi(\bar{X})=0$ corresponds to the cylinder, whose interior $X$ is uniformized by the cyclic elementary group $\Gamma=\langle\gamma\rangle$, with a unique closed geodesic of length $\ell$, the translation length of the generator $\gamma$. In other words, $X$ is conformal to the hyperbolic cylinder $H_{\ell}=$ $\left(\mathbb{R}_{r} \times(\mathbb{R} / \mathbb{Z})_{t}, g=d r^{2}+\cosh ^{2}(r) \ell^{2} d t^{2}\right)$ where $\ell>0$ is the length of the unique closed geodesic $\{r=0\}$. By Theorem 2.2 it suffices to compute it for the conformal representative of the boundary at infinity $\left.\left(|\sinh (r)|^{-2} g\right)\right|_{T \partial \bar{X}}=\ell^{2} d t^{2}$ and the result will be given by multiplying by $\ell_{h_{0}}(\partial \bar{X}) / 2 \ell$. The scattering matrix for the conformal representative $\ell^{2} d t^{2}$ is computed in [15], it is decomposable on the Fourier modes, the solution of (2.4) for data $f=1$ on the boundary is

$$
\begin{aligned}
u_{\lambda}(r, t)= & \left.|\sinh r|^{\lambda-1} F\left(\frac{1-\lambda}{2}, 1-\frac{\lambda}{2}, \frac{3}{2}-\lambda ;-\sinh ^{-2}(r)\right)\right) \\
& +\left(\frac{\Gamma(\lambda / 2)}{\Gamma((1-\lambda) / 2)}\right)^{2} \frac{\Gamma\left(\frac{1}{2}-\lambda\right)}{\Gamma\left(\lambda-\frac{1}{2}\right)}|\sinh r|^{-\lambda} F\left(\frac{\lambda}{2}, \frac{\lambda+1}{2}, \lambda+\frac{1}{2} ;-\sinh ^{-2}(r)\right)
\end{aligned}
$$

where $F$ is the hypergeometric function but since here we chose $x=|\sinh r|^{-1}$ (to have the right conformal representative on $\partial \bar{X})$ and since $F(a, b, c ; 0)=1$, this gives easily $\mathcal{S}(\lambda) 1$ :

$$
\mathcal{S}(\lambda) 1=2^{2 \lambda-1}\left(\frac{\Gamma(\lambda / 2)}{\Gamma((1-\lambda) / 2)}\right)^{2}=\frac{\pi}{2}(1-\lambda)^{2}+O\left((1-\lambda)^{3}\right), \quad \lambda \rightarrow 1 .
$$

We can thus do the same reasoning as above, but now $\nu_{1}=2, w_{\lambda}=w_{1}$ and $\beta=\pi / 2$, we finally get, for the conformal representative $\ell^{2} d t^{2}$,

$$
\operatorname{det}^{\prime}(\mathcal{S}(1))=\lim _{\lambda \rightarrow 1} \frac{\operatorname{det} \mathcal{S}(\lambda)}{\pi(\lambda-1)^{2} / 2}=\frac{2}{\pi} \lim _{\lambda \rightarrow 1} \frac{R_{\Gamma}(1-\lambda)}{(1-\lambda)^{2}}=\frac{2 \ell^{2}}{\pi}
$$

where the Selberg zeta function for this special case in the functional equation (2.12) is $Z_{\Gamma}(\lambda)=$ $\prod_{k \in \mathbb{N}_{0}}\left(1-e^{-(\lambda+k) \ell}\right)^{2}$ (see Prop 3.3 of Patterson [30]), thus here $R_{\Gamma}(\lambda)=\left(1-e^{-\lambda \ell}\right)^{2}$. This gives the proof. For completeness, we will give another explicit computation of this case in the Appendix using the flat annulus conformal to the hyperbolic cylinder $H_{\ell}$.

We remark that in the proof above, the fact that $\nu_{1}=1$ when $\chi(\bar{X})<0$ shows that $\operatorname{det} \mathcal{S}(\lambda)$ has a zero of order exactly 1 at $\lambda=1$ and from (2.12) we deduce that $Z_{\Gamma}(\lambda)$ has a zero of order exactly $-\chi(\bar{X})+1$, as well as the Ruelle function $R_{\Gamma}(\lambda)$. It also implies that $\nu_{0}=-1$ since $\nu_{\lambda_{0}}=-\nu_{1-\lambda_{0}}$ for any $\lambda_{0} \in \mathbb{C}$ (see comments after equation 1.1 of [31]). Then, using also that $\lambda=1$ is not a pole of the resolvent $7 R(\lambda):=(\Delta-\lambda(1-\lambda))^{-1}$ since 0 is not an $L^{2}$ eigenvalue of $\Delta_{g}$, we deduce from Theorem 1.1 of [18] that $\lambda=0$ is a pole of order 1 , with residue of rank 1 , of the meromorphic extension of $R(\lambda)$ to $\mathbb{C}$.

\section{Computation of $\operatorname{det}^{\prime}(\mathcal{N})$ Using Osgood-Phillips-Sarnak uniformization}

In [29], Osgood, Phillips and Sarnak (see also Brendle [3]) proved that in each conformal class of metrics on a compact surface with boundary,

- there is a unique representative which is flat, with constant geodesic curvature boundary,

- there is a unique representative which has constant curvature and totally geodesic boundary.

\footnotetext{
${ }^{7}$ The resolvent $R(\lambda)$ is an analytic family of operators acting on $L^{2}(X)$ if $\Re(\lambda)>1$, it admits a meromorphic continuation to $\lambda \in \mathbb{C}$ as an operator mapping $C_{0}^{\infty}(X)$ to $C^{\infty}(X)$ by a result of Guillopé-Zworski [20].
} 
The flat uniformization has been used by Edward-Wu [7] to $\operatorname{compute} \operatorname{det}^{\prime}(\mathcal{N})$ for a topological disc (i.e. the case $\chi(\bar{X})=1$ ), they found the same result than in our Theorem 1.2 . One can do the same for a topological cylinder, it is uniformized as a flat annulus $\{z \in \mathbb{C} ; 1 \leq|z| \leq \rho\}$ for some $\rho>1$ and it is possible to compute $\operatorname{det}^{\prime}(\mathcal{N})$, we do the calculation in the Appendix and show that it fits with the value in Theorem 1.2. If $\chi(\bar{X})<0$, there does not seem to be apparent way to express $\operatorname{det}^{\prime}(\mathcal{N})$ in terms of geometric invariants. Thus, we use the constant curvature uniformization with geodesic boundary, in this case one obtains a compact hyperbolic surface with geodesic boundary $(\bar{X}, \bar{g})$. The surface $\bar{X}$ is uniformized so that $\bar{X}$ is isometric to $G_{0} \backslash \mathbb{H}^{2}$ where $G_{0}$ is a group of isometries of $\mathbb{H}^{2}$, containing some symmetries. Associated to $G_{0}$, there is a natural Selberg type zeta function $Z_{G_{0}}(\lambda)$ [19], defined in (1.4). Now, let $M=\bar{X} \sqcup \bar{X}$ be the manifold obtained by gluing two copies of $\bar{X}$ at the boundary $\partial \bar{X}$, then $M$ has smooth structure of surface with no boundary such that the natural involution is smooth. We can extend the hyperbolic metric $\bar{g}$ on $M$ by symmetry and the new metric, called $g$, is smooth on $M$ since the structure of the metric $\bar{g}$ in Fermi coordinates $(r, t) \in[0, \epsilon) \times(\mathbb{R} / \mathbb{Z})$ near each connected component $C=\{r=0\}$ of $\partial \bar{X}$ is

$$
\bar{g}=d r^{2}+\ell^{2} \cosh ^{2}(t) d t^{2}
$$

for $\ell>0$ the length of $C$. The manifold $M$ is isometric to the quotient $G \backslash \mathbb{H}^{2}$ of the hyperbolic plane by the co-compact Fuchsian group $G=G_{0} \cap \operatorname{Isom}^{+}\left(\mathbb{H}^{2}\right)$, the subgroup of index 2 of direct isometries of $G_{0}$, we will call $(M, g)$ the double of $(\bar{X}, \bar{g})$. Let us denote by $V:=\partial \bar{X}$ the boundary of $\bar{X}$, the manifold $M \backslash V$ can be compactified canonically so that it corresponds to two connected components isometric to $\bar{X}$, we will consider this manifold and will denote it $\bar{X}^{2}$ by abuse of notation. We show

Theorem 3.1. Let $(\bar{X}, \bar{g})$ be an oriented surface with boundary with Euler characteristic $\chi(\bar{X})<$ 0 . Let $G_{0}$ be the discrete subgroup of $\operatorname{Isom}\left(\mathbb{H}^{2}\right)$ such that $(\bar{X}, \bar{g})$ is conformal to the hyperbolic surface with geodesic boundary $G_{0} \backslash \mathbb{H}^{2}$ and let $G:=G_{0} \cap \mathrm{Isom}^{+}\left(\mathbb{H}^{2}\right)$ be the index 2 subgroup of orientation preserving elements of $G_{0}$, so that $G \backslash \mathbb{H}^{2}$ is the closed hyperbolic surface realized by doubling $G_{0} \backslash \mathbb{H}^{2}$ along the boundary. Then

$$
\frac{\operatorname{det}^{\prime}(\mathcal{N})}{\ell(\partial \bar{X})}=-\frac{Z_{G}^{\prime}(1)}{\left(Z_{G_{0}}(1)\right)^{2}} \frac{e^{\ell(\partial \bar{X}) / 4}}{2 \pi \chi(\bar{X})}
$$

where $Z_{G}(\lambda), Z_{G_{0}}(\lambda)$ are the Selberg zeta function associated respectively to the group $G, G_{0}$, and defined respectively in (1.2) and (1.4).

Proof: By conformal invariance of $\operatorname{det}^{\prime}(\mathcal{N}) / \ell(\partial \bar{X})$ and using the constant negative curvature with totally geodesic boundary uniformization, it suffices to assume that $\bar{X}=G_{0} \backslash \mathbb{H}^{2}$ as above. Following the notation preceding the Theorem, we let $M=G \backslash \mathbb{H}^{2}$ be the double of $\bar{X}$ where $G=G_{0} \cap \operatorname{Isom}^{+}\left(\mathbb{H}^{2}\right)$. We denote by $\Delta_{\bar{X}^{2}}$ the Laplacian on $M$ with Dirichlet condition on the geodesic boundary $\partial \bar{X}$, that is the direct sum $\Delta_{\bar{X}} \oplus \Delta_{\bar{X}}$ on the two copies of $\bar{X}$ in $M$ where $\Delta_{\bar{X}}$ is the Dirichlet realization of the Laplacian on $\bar{X}$, then its spectrum is clearly the same than $\Delta_{\bar{X}}$ but with double the multiplicity and thus

$$
\operatorname{det}\left(\Delta_{\bar{X}^{2}}\right)=\left(\operatorname{det}\left(\Delta_{\bar{X}}\right)\right)^{2}
$$

From the proof of Theorem $B^{*}$ of Burghelea-Friedlander-Kappeler 4, we get that, if $\Delta_{M}$ is the Laplacian on $(M, g)$

$$
\frac{\operatorname{det}^{\prime}\left(\Delta_{M}\right)}{\operatorname{det}\left(\Delta_{\bar{X}^{2}}\right)}=\frac{\operatorname{vol}(M)}{2 \ell(\partial \bar{X})} \operatorname{det}^{\prime}(\mathcal{N})
$$

where $\mathcal{N}$ is the Dirichlet-to-Neumann map on $\partial \bar{X}$ defined in the Introduction for either copy of $\bar{X}$ in $M, \operatorname{vol}(M)$ is the volume of $M$ for the hyperbolic metric $g$ (i.e. $-4 \pi \chi(\bar{X})$ by GaussBonnet formula), $\ell(\partial \bar{X})$ is the length of the geodesic boundary $\partial \bar{X}$. But from Sarnak 34 ] (see also D'Hoker-Phong [6], Voros [37]) we have (recall $\chi(M)=2 \chi(\bar{X})$ )

$$
\operatorname{det}^{\prime}\left(\Delta_{M}\right)=Z_{G}^{\prime}(1) e^{-2 \eta \chi(\bar{X})}, \quad \eta=2 \zeta^{\prime}(-1)-\frac{1}{4}+\frac{1}{2} \log (2 \pi)
$$


with $\zeta$ the Riemann zeta function.

We now need to compute the determinant $\operatorname{det}\left(\Delta_{\bar{X}}\right)$ of the Dirichlet Laplacian on a hyperbolic surface with geodesic boundary $\bar{X}$, using the Selberg function $Z_{G_{0}}(\lambda)$. This can be done by methods of Sarnak [34] and a trace formula by Guillopé [19, Prop 3.1], we show the

Proposition 3.2. If $\bar{X}=G_{0} \backslash \mathbb{H}^{2}$ is a hyperbolic surface with geodesic boundary $\partial \bar{X}$ of length $\ell(\partial \bar{X})$ and $\Delta_{\bar{X}}$ is the Dirichlet Laplacian on $\bar{X}$, then

$$
\operatorname{det}\left(\Delta_{\bar{X}}-\lambda(1-\lambda)\right)=Z_{G_{0}}(\lambda)\left(e^{\eta-\frac{\ell(\partial \bar{X})}{8 \chi(X)}(1-2 \lambda)+\lambda(1-\lambda)} \frac{(2 \pi)^{\lambda-1}}{G(\lambda)^{2} \Gamma(\lambda)}\right)^{-\chi(\bar{X})},
$$

where $\chi(\bar{X})$ is the Euler characteristic of $\bar{X}, G(\lambda)$ is Barnes' function, $Z_{G_{0}}(\lambda)$ is the Selberg zeta function of (1.4) and $\eta$ is the constant defined in (3.3).

Proof: It suffices to apply the proof of Sarnak [34] (done in the case with no boundary) to the trace formula obtained in Proposition 3.1 of [19]

$$
\begin{aligned}
\frac{Z_{G_{0}}^{\prime}(\lambda)}{(2 \lambda-1) Z_{G_{0}}(\lambda)}= & \operatorname{Tr}\left(R_{\bar{X}}(\lambda)-R_{\bar{X}}\left(\lambda_{0}\right)\right)+\frac{Z_{G_{0}}^{\prime}\left(\lambda_{0}\right)}{\left(2 \lambda_{0}-1\right) Z_{G_{0}}\left(\lambda_{0}\right)} \\
& -\chi(\bar{X})\left(\frac{\Gamma^{\prime}(\lambda)}{\Gamma(\lambda)}-\frac{\Gamma^{\prime}\left(\lambda_{0}\right)}{\Gamma\left(\lambda_{0}\right)}\right)-\frac{\ell(\partial \bar{X})}{4}\left(\frac{1}{2 \lambda-1}-\frac{1}{2 \lambda_{0}-1}\right),
\end{aligned}
$$

here $R_{\bar{X}}(\lambda):=\left(\Delta_{\bar{X}}-\lambda(1-\lambda)\right)^{-1}$. Indeed one deduces from this the formula

$$
\operatorname{det}\left(\Delta_{\bar{X}}-\lambda(1-\lambda)\right)=Z_{G_{0}}(\lambda) e^{-\frac{\ell(\partial \bar{X})}{4} \lambda+C \lambda(1-\lambda)+D}\left(\frac{\left(\Gamma_{2}(\lambda)\right)^{2}(2 \pi)^{\lambda}}{\Gamma(\lambda)}\right)^{-\chi(\bar{X})}
$$

for some constants $C, D$ and where the digamma function $\Gamma_{2}$ is the inverse of the Barnes function $G$ used in (2.12). To compute the constants, we consider the asymptotics as $\lambda \rightarrow+\infty$ of this identity. First we get an asymptotic for the left hand side through the use of the heat kernel small time asymptotic as in 34:

$$
\begin{aligned}
\log \left(\operatorname{det}\left(\Delta_{\bar{X}}-\lambda(1-\lambda)\right)\right)= & -a_{1} \lambda(\lambda-1) \log (\lambda(\lambda-1))+a_{1} \lambda(\lambda-1)+2 \sqrt{\pi} a_{2}\left(\lambda-\frac{1}{2}\right) \\
& +a_{3} \log (\lambda(\lambda-1))+o(1) \\
= & -a_{1} \lambda^{2} \log \lambda+a_{1} \lambda^{2}+2 a_{1} \lambda \log \lambda+2 \sqrt{\pi} a_{2} \lambda+2 a_{3} \log \lambda \\
& -\sqrt{\pi} a_{2}-\frac{1}{2} a_{1}+o(1)
\end{aligned}
$$

where $a_{1}, a_{2}, a_{3}$ are the heat invariant obtained in [29, Appendix]

$$
\operatorname{Tr}\left(e^{-t \Delta_{\bar{X}}}\right)=t^{-1}\left(a_{1}+a_{2} t^{\frac{1}{2}}+a_{3} t\right)+o(1)=\left(-\frac{1}{2} \chi(\bar{X})-\frac{\ell(\partial \bar{X})}{8 \sqrt{\pi}} t^{\frac{1}{2}}+\frac{\chi(\bar{X})}{6} t\right)+o(1)
$$

as $t \rightarrow 0$. On the other hand, using Stirling formula and $\lim _{\lambda \rightarrow+\infty} Z_{G_{0}}(\lambda)=1$ in the right hand side of (3.4), we get

$$
\begin{aligned}
2 \log \left(\operatorname{det}\left(\Delta_{\bar{X}}-\lambda(1-\lambda)\right)\right)= & \chi(\bar{X})\left(-(\lambda-1)^{2} \log (\lambda-1)+\frac{3}{2}(\lambda-1)^{2}-\lambda \log \lambda+\lambda\right. \\
& \left.+\frac{2}{3} \log \lambda+\log (2 \pi)-2 \zeta^{\prime}(-1)\right)+C \lambda(1-\lambda)+D-\frac{\ell(\partial \bar{X})}{4} \lambda+o(1) \\
= & -\chi(\bar{X})\left(-\lambda^{2} \log \lambda+\frac{3}{2} \lambda^{2}+\lambda \log \lambda-\lambda-\frac{1}{3} \log \lambda+\frac{1}{2} \log (2 \pi)\right. \\
& \left.-2 \zeta^{\prime}(-1)\right)-C \lambda^{2}+\left(C-\frac{\ell(\partial \bar{X})}{4}\right) \lambda+D+o(1) .
\end{aligned}
$$

Identifying the coefficient gives

$$
C=-\chi(\bar{X}), \quad D=\chi(\bar{X})\left(\frac{1}{2} \log (2 \pi)-2 \zeta^{\prime}(-1)+\frac{1}{4}\right)+\frac{\ell(\partial \bar{X})}{8}
$$


and this gives the desired formula.

The Theorem 3.1 is now proved by combining (3.2), (3.3), (3.1) and Proposition 3.2 with $\lambda=1$ (note that $Z_{G_{0}}(\lambda)$ is holomorphic with no zero near $\lambda=1$, as $\Delta_{\bar{X}}$ has no zero eigenvalue).

Observe that $\operatorname{det}\left(\Delta_{\bar{X}}-\lambda(1-\lambda)\right)^{2}=\operatorname{det}\left(\Delta_{\bar{X}^{2}}-\lambda(1-\lambda)\right)$ where $\bar{X}^{2}$ is two disjoint copies of $\bar{X}$ and $\Delta_{\bar{X}^{2}}=\Delta_{\bar{X}} \oplus \Delta_{\bar{X}}$ the Dirichlet Laplacian. Similarly one has $\chi(M)=2 \chi(\bar{X})$ thus one can use Sarnak's formula 34 to get

$$
\frac{\operatorname{det}\left(\Delta_{M}-\lambda(1-\lambda)\right)}{\operatorname{det}\left(\Delta_{\bar{X}^{2}}-\lambda(1-\lambda)\right)}=\frac{Z_{G}(\lambda)}{Z_{G_{0}}(\lambda)^{2}} e^{-\frac{\ell(\partial \bar{X})}{4}(1-2 \lambda)} .
$$

Moreover, if $\Gamma \backslash \mathbb{H}^{2}$ is the Mazzeo-Taylor uniformization of $\bar{X}$, then Proposition 3.2 and Theorem 3.1 together give the formula

$$
\left[\lambda^{\chi(\bar{X})-1} R_{\Gamma}(\lambda)\right]_{\mid \lambda=0}=-\frac{Z_{G}^{\prime}(1)}{Z_{G_{0}}(1)^{2}} e^{\frac{\ell(\partial \bar{X})}{4}}(2 \pi)^{-\chi(\bar{X})} .
$$

This formula relates in a regularized way the the length spectrum of $\bar{X}$ and that of the noncompact uniformization $\Gamma \subset \mathbb{H}^{2}$. It does not appear obvious to us how to obtain any relation between these spectrum by other methods.

\section{Dirichlet-to-Neumann for Yamabe operator}

We discuss now what is the higher dimensional version of Lemma 2.1.

First, let $(X, g)$ an asymptotically hyperbolic manifold with constant scalar curvature equal to $\mathrm{Scal}_{g}=-n(n+1)$. From Graham-Lee 14, there is boundary defining function $x$ such that the metric near the boundary is $\left(d x^{2}+h(x)\right) / x^{2}$ for some 1-parameter family of metric $h(x)$ on $\partial \bar{X}$. A straightforward computation gives

$$
\mathrm{Scal}_{g}=-n(n+1)=-n(n+1)+n x \partial_{x} \log (\operatorname{det} h(x))+x^{2} \operatorname{Scal}_{\bar{g}}
$$

which implies that $\operatorname{tr}_{h_{0}}\left(h_{1}\right)=0$ if $h_{0}=h(0)$ and $h_{1}=\partial_{x} h(0)$. The Poisson problem (2.2) with initial data $f \in C^{\infty}(\partial \bar{X})$ can then be solved at $\lambda=(n+1) / 2$ by results of [15, 16] (for such $\lambda$, we do not need full evenness of $g$ but only $\left.\operatorname{tr}_{h_{0}}\left(h_{1}\right)\right)$. Since

$$
\left(\Delta_{g}-\frac{n+1}{2} \cdot \frac{n-1}{2}\right) \hat{\rho}^{\frac{n-1}{2}} u=0, \quad u \in C^{\infty}(\bar{X}),\left.\quad u\right|_{\partial \bar{X}}=f
$$

is equivalent to solve the elliptic Dirichlet problem

$$
\left(\Delta_{\bar{g}}+\operatorname{Scal}_{\bar{g}} \frac{n-1}{4 n}\right) u=0,\left.\quad u\right|_{\partial \bar{X}}=f
$$

for $\bar{g}=x^{2} g$ by conformal covariance of the Yamabe operator, and since $\partial_{n}=\partial_{x}$ at the boundary, we deduce that $\mathcal{S}((n+1) / 2)=\mathcal{N}$ where $\mathcal{N}$ is the DN map for the conformal Laplacian of $(\bar{X}, \bar{g})$ and $\mathcal{S}(\lambda)$ is the scattering operator for $(X, g)$ with boundary defining function. Remark that $\partial \bar{X}$ is a minimal hypersurface of $(\bar{X}, \bar{g})$ since $h_{1}=0$.

Conversely, let $(\bar{X}, \bar{g})$ be an $(n+1)$-dimensional smooth compact Riemannian manifold with boundary, then it is proved by Aviles-Mac Owen [2] that there exists a complete metric $g_{0}$ conformal to $\bar{g}$ on the interior $X$ and with negative constant scalar curvature $\operatorname{Scal}_{g_{0}}=-n(n+1)$. Moreover it is proved by Andersson-Chruściel-Friedrich [1, Th. 1.3] (see also Mazzeo [25]) that $g_{0}$ is asymptotically hyperbolic with log terms in the expansion, more precisely let $\rho$ be a geodesic boundary defining function of $\partial \bar{X}$ for $\bar{g}$, i.e. $\bar{g}=d \rho^{2}+\bar{h}(\rho)$ for some 1 parameter family of metric $\bar{h}(\rho)$ on $\partial \bar{X}$, we have

$$
g_{0}=\frac{\bar{g}\left(1+\rho v+\rho^{n} w\right)}{\rho^{2}}=\frac{\bar{g}}{\hat{\rho}^{2}}
$$


with $v \in C^{\infty}(\bar{X}), w \in C^{\infty}(X)$ and $w$ having a polyhomogenous expansion

$$
w(\rho, y) \sim \sum_{i=0}^{\infty} \sum_{j=0}^{N_{i}} u_{i j} \rho^{i}(\log \rho)^{j}
$$

near the boundary, $N_{i} \in \mathbb{N}_{0}$ and $u_{i j} \in C^{\infty}(\partial \bar{X})$. Note that by Graham-Lee Lemma [14], there exists for $h_{0}:=\bar{h}(0)=\left.\bar{g}\right|_{T \partial \bar{X}}$ a boundary defining function $x=\rho+O\left(\rho^{2}\right)$ such that $g_{0}=\left(d x^{2}+h(x)\right) / x^{2}$ near $\partial \bar{X}$ with $h(x)$ a 1-parameter family of metrics on $\partial \bar{X}$ such that $h(0)=h_{0}$, with the regularity of $\rho v+\rho^{n} w$. We denote by $\overline{g_{0}}=x^{2} g_{0}$ and as before $\operatorname{Tr}_{h_{0}}\left(h_{1}\right)=0$ if $h(x)=h_{0}+x h_{1}+O\left(x^{2}\right)$ (i.e. $h_{1}$ is the second fundamental form of $\bar{g}_{0}$ ). Then we can consider the elliptic Dirichlet problem (4.1) where $f \in C^{\infty}(\bar{X})$ is fixed. It has a unique solution $u \in C^{\infty}(\bar{X})$ which allows to define $\mathcal{N}: C^{\infty}(\partial \bar{X}) \rightarrow C^{\infty}(\partial \bar{X})$ by

$$
\mathcal{N} f=-\left.\partial_{n} u\right|_{\partial \bar{X}}
$$

where $u$ is the solution of (4.1) and $\partial_{n}=\partial_{\rho}=\partial_{x}$ the interior unit normal vector field to $\partial \bar{X}$. An easy computation as above shows that (4.1) is equivalent to solving

$$
\left(\Delta_{g_{0}}-\frac{n+1}{2} \cdot \frac{n-1}{2}\right) \hat{\rho}^{\frac{n-1}{2}} u=0, \quad u \in C^{\infty}(\bar{X}),\left.\quad u\right|_{\partial \bar{X}}=f .
$$

by conformal covariance of the Yamabe operator. But this is exactly the Poisson problem at energy $\lambda=(n+1) / 2$ for the asymptotically hyperbolic manifold $g_{0}$, dealt with 8 by GrahamZworski [15. Since $\partial_{n}=\partial_{x}$ we thus deduce that

$$
\mathcal{N} f=-S\left(\frac{n+1}{2}\right) f+\frac{(n-1) \omega}{2} f
$$

where $\hat{\rho}=x\left(1+x \omega+O\left(x^{2}\right)\right)$ for some $\omega \in C^{\infty}(\partial \bar{X})$. But $\bar{g} x^{2}=\hat{\rho}^{2} \bar{g}_{0}$ thus applying to any vector field $V \in \partial \bar{X}$, this gives

$$
\left(h_{0}(V)+x h_{1}(V)\right)(1-2 x \omega)=\left(h_{0}(V)+x \bar{h}_{1}(V)\right)+O\left(x^{2}\right)
$$

where $\bar{h}_{1}=\partial_{\rho} \bar{h}(0)$ is the second fundamental form of $\bar{g}$, this implies clearly that $\bar{h}_{1}=h_{1}-2 \omega h_{0}$ and, taking the trace with respect to metric $h_{0}$, we get $\omega=-\frac{1}{2 n} \operatorname{Tr}_{h_{0}}\left(\bar{h}_{1}\right)$. We have thus proved

Proposition 4.1. The Dirichlet-to-Neumann map for the conformal Laplacian of $\bar{g}$ is

$$
\mathcal{N}=\mathcal{S}\left(\frac{n+1}{2}\right)-\frac{(n-1)}{2} K
$$

where $K=\operatorname{Tr}_{h_{0}}\left(\bar{h}_{1}\right) / 2 n$ is the mean curvature of $\partial \bar{X}$ for $\bar{g}, \mathcal{S}(\lambda)$ is the scattering operator associated to the complete metric with constant scalar curvature metric $g_{0}$, conformal to $\bar{g}$, and for choice of conformal representative $h_{0}=\left.\bar{g}\right|_{T \partial \bar{X}}$.

Another consequence, if $(\bar{X}, \bar{g})$ is conformal to a convex co-compact quotient $\Gamma \backslash \mathbb{H}^{n+1}$ in even dimension, the determinant of $\mathcal{N}+(n-1) K / 2$ can be obtained by the functional equation of [17] in terms of a special values of Selberg zeta function of $\Gamma$. We do not write the details and refer the reader to that paper, since this is much less interesting than for surfaces.

\footnotetext{
${ }^{8}$ They actually study it for smooth asymptotically hyperbolic manifolds but their proof works as well when log-terms enter the expansion of the metric at the boundary, in particular here the first log terms appear at order $x^{n} \log (x)$, thus they do not change the form of the two first asymptotic terms in the solution of the Poisson problem at energy $(n+1) / 2$ : one has

$$
\hat{\rho}^{\frac{n-1}{2}} u \sim x^{\frac{n-1}{2}}\left(f+O\left(x^{2}\right)\right)+x^{\frac{n+1}{2}}\left(-\mathcal{S}\left(\frac{n+1}{2}\right) f+O(x)\right)
$$

where $\mathcal{S}(\lambda)$ is the scattering operator (see [15]), the fact that there is no $x^{\frac{n+1}{2}} \log x$ terms and no other terms than $x^{\frac{n+1}{2}} \mathcal{S}((n+1) / 2) f$ at order $x^{\frac{n+1}{2}}$ is because $\operatorname{Tr}_{h_{0}}\left(h_{1}\right)=0$ (then $\mathcal{S}(\lambda)$ has no residue at $\left.(n+1) / 2\right)$, see Lemma 4.1 of [16] for more details.
} 


\section{Appendix: THE CYlinder}

As a particular case of [26], a smooth surface with boundary $\bar{X}$, with Euler characteristic $\chi(\bar{X})=0$, is conformal to a hyperbolic cylinder $\langle\gamma\rangle \backslash \mathbb{H}^{2}$ with $\gamma: z \rightarrow e^{\ell} z$, which himself is conformal to the flat annulus $A_{\rho}:=\{z \in \mathbb{C} ; 1<|z|<\rho\}$ with $\rho:=e^{2 \pi^{2} / \ell}$, a conformal diffeomorphism being induced by the map

$$
U: z \in \mathbb{H}^{2} \rightarrow e^{2 i \pi(\log z) / \ell+2 \pi^{2} / \ell} .
$$

satisfying $U(z)=U\left(e^{\ell} z\right)$.

We compute in this appendix the determinant of the Dirichlet-to-Neumann map for the annulus $\overline{A_{\rho}}$ and check that if fits with the value found by the technic of the functional equation of Selberg zeta function used above, giving an alternative way of computing $\operatorname{det}^{\prime}(\mathcal{N})$ for the cylinder, much in the spirit of [7].

Polar coordinates $z=r e^{\mathrm{i} \theta}$ induce Fourier mode decompositions

$$
f=\left(f_{\rho}, f_{1}\right) \in L^{2}\left(\partial A_{\rho}\right) \simeq L^{2}(\mathbb{R} / 2 \pi \mathbb{Z}) \otimes \mathbb{C}^{2} \rightarrow\left(\widehat{f}_{\rho}(n), \widehat{f}_{1}(n)\right)_{n \in \mathbb{Z}} \in \bigoplus_{n \in \mathbb{Z}} F_{n}
$$

with $F_{n} \simeq \mathbb{C}^{2}$ and the DN map $\mathcal{N} \simeq \bigoplus_{n \in \mathbb{Z}} \mathcal{N}_{n}$ is diagonal with respect to this Fourier decomposition. The harmonic functions $h_{0}$ and $h_{1}$ defined on $A_{\rho}$ by

$$
h_{0}(z)=1, \quad h_{1}(z)=1-(1+\rho) \ln |z| /(\rho \ln \rho), \quad z \in A_{\rho}
$$

give eigenvectors of the DN map on $F_{0}$ : $(1,1)$ and $\left(-\rho^{-1}, 1\right)$ with respective eigenvalues 0 and $(1+\rho) /(\rho \ln \rho)$.

For $n \in \mathbb{Z}^{*}$, the harmonic functions

$$
h_{\rho, n}(z)=\frac{z^{n}-\bar{z}^{-n}}{\rho^{n}-\rho^{-n}}, \quad h_{1, n}(z)=\frac{\rho^{n} \bar{z}^{-n}-\rho^{-n} z^{n}}{\rho^{n}-\rho^{-n}}
$$

have traces on $\partial A_{\rho}$ inducing the canonical base of $F_{n} \simeq \mathbb{C}^{2}$

$$
h_{\rho, n}(z)=\left\{\begin{array}{ll}
e^{\mathrm{i} n \theta} & \text { if }|z|=\rho \\
0 & \text { if }|z|=1
\end{array}, \quad h_{1, n}(z)= \begin{cases}0 & \text { if }|z|=\rho \\
e^{\mathrm{i} n \theta} & \text { if }|z|=1\end{cases}\right.
$$

and their derivatives under the radial vector field $\partial_{r}$

$$
\partial_{r} h_{\rho, n}\left(r e^{\mathrm{i} n \theta}\right)=n \frac{r^{n-1}+r^{-n-1}}{\rho^{n}-\rho^{-n}} e^{\mathrm{i} n \theta}, \quad \partial_{r} h_{1, n}\left(r e^{\mathrm{i} n \theta}\right)=-n \frac{\rho^{n} r^{-n-1}+\rho^{-n} r^{n-1}}{\rho^{n}-\rho^{-n}} e^{\mathrm{i} n \theta}
$$

give the matrix $\mathcal{N}_{n}$ with respect to the canonical base of $F_{n}$. Observing that the interior normal derivative $\partial_{n}$ is $-\partial_{r}$ on $\{|z|=\rho\}$ and its opposite $\partial_{r}$ on $\{|z|=1\}$, we have, with $\alpha=\log \rho$,

$$
\mathcal{N}_{n}=\left(\begin{array}{cc}
n \rho^{-1} \frac{\rho^{n}+\rho^{-n}}{\rho^{n}-\rho^{-n}} & -\frac{2 n \rho^{-1}}{\rho^{n}-\rho^{-n}} \\
-\frac{2 n}{\rho^{n}-\rho^{-n}} & n \frac{\rho^{n}+\rho^{-n}}{\rho^{n}-\rho^{-n}}
\end{array}\right)=\frac{n}{\sinh (n \alpha)}\left(\begin{array}{cc}
\mathrm{e}^{-\alpha} \cosh (n \alpha) & -\mathrm{e}^{-\alpha} \\
-1 & \cosh (n \alpha)
\end{array}\right)
$$

with determinant $\delta_{n}=n^{2} \mathrm{e}^{-\alpha}$ and eigenvalues

$$
\lambda_{n, \pm}=\frac{\mathrm{e}^{-\alpha / 2} n}{\sinh (n \alpha)} \cosh (\alpha / 2) \cosh (n \alpha) \pm \sqrt{\sinh ^{2}(\alpha / 2) \cosh ^{2}(n \alpha)+1}
$$

The following lemma claims the product relation " $\prod \lambda_{n,+} \prod \lambda_{n,-}=\prod \delta_{n}$ ":

Lemma 5.1. Let $u=\left(u_{n}\right)_{n \geq 1}$ a sequence with positive terms such that $u_{n}=n^{k}\left(1+\varepsilon_{n}\right)$ with $\varepsilon_{n}=O\left(\mathrm{e}^{-a n}\right)$ for some non negative $k$ and $a$. If $\zeta_{u}$ is the zeta function $\zeta_{u}(s)=\sum_{n \geq 1} u_{n}^{-s}$ convergent for $\Re s>k^{-1}$, then $\zeta_{u}$ extends meromorphically to the complex plane and is regular for $s=0$ with

$$
\zeta_{u}(0)=\zeta(0), \quad \partial_{s} \zeta_{u}(0)=k \zeta^{\prime}(0)-\sum_{n \geq 1} \ln \left(1+\varepsilon_{n}\right)
$$


where $\zeta$ is the Riemann zeta function. If $u, v$ are two such sequences, then the sequence $w$ defined by $w_{n}=u_{n} v_{n}, n \geq 1$ is again of the same type and $\partial_{s} \zeta_{u}(0)+\partial_{s} \zeta_{v}(0)=\partial_{s} \zeta_{w}(0)$.

Proof: Let $G(s, \varepsilon)$ be the function defined for $\varepsilon$ small and $s \in \mathbb{C}$, holomorphic in $s$, such that

$$
(1+\varepsilon)^{-s}=1-s G(s, \varepsilon), \quad|G(s, \varepsilon)|+\left|\partial_{s} G(s, \varepsilon)\right|={ }_{s, \varepsilon \sim 0} O(\varepsilon), \quad G(s, \varepsilon)_{\mid s=0}=\ln (1+\varepsilon) .
$$

We have then

$$
\zeta_{u}(s)=\sum_{n \geq 1}\left(n^{k}\left(1+\varepsilon_{n}\right)\right)^{-s}=\sum_{n \geq 1} n^{-k s}-s \sum_{n \geq 1} n^{-k s} G\left(s, \varepsilon_{n}\right)=\zeta(k s)-s \sum_{n \geq 1} n^{-k s} G\left(s, \varepsilon_{n}\right)
$$

and

$$
\zeta_{u}(0)=\zeta(0), \quad \partial_{s} \zeta_{u}(0)=k \zeta^{\prime}(0)-\sum_{n \geq 1} G\left(0, \varepsilon_{n}\right)=k \zeta^{\prime}(0)-\sum_{n \geq 1} \ln \left(1+\varepsilon_{n}\right) .
$$

The $\zeta$-regularized determinant $\operatorname{det}^{\prime} \mathcal{N}$ for the DN map on the annulus $\overline{A_{\rho}}$ is defined through the zeta function

$$
\zeta_{\rho}(s)=[(1+\rho) /(\rho \ln \rho)]^{-s}+\sum_{n \in \mathbb{Z}^{*}}\left[\lambda_{-, n}^{-s}+\lambda_{+, n}^{-s}\right] .
$$

According to the preceding Lemma, if $\widetilde{\zeta}_{\rho}$ is the zeta function defined by $\widetilde{\zeta}_{\rho}(s)=[(1+\rho) /(\rho \ln \rho)]^{-s}+$ $2 \sum_{n \geq 1} \delta_{n}^{-s}$. We have

$$
\partial_{s} \zeta_{\rho}(0)=\partial_{s} \widetilde{\zeta}_{\rho}(0)=-\ln [(1+\rho) /(\rho \ln \rho)]+2 \alpha \zeta(0)+4 \zeta^{\prime}(0)=-\ln [(1+\rho) /(\ln \rho)]-2 \ln (2 \pi),
$$

where we have used $\lambda_{n,+} \lambda_{n,-}=\delta_{|n|}=n^{2} e^{-\alpha}$ (so that the $\log \left(1+\epsilon_{n}\right)$ terms disappear in the Lemma) and for the last equality $\zeta(0)=-1 / 2$ and $\zeta^{\prime}(0)=-\ln (2 \pi) / 2$. Hence

$$
\frac{\operatorname{det}^{\prime} \mathcal{N}}{\ell\left(\partial \overline{A_{\rho}}\right)}=\frac{\mathrm{e}^{-\partial_{s} \zeta_{\rho}(0)}}{2 \pi(1+\rho)}=\frac{2 \pi}{\ln \rho}=\frac{\ell}{\pi}
$$

which perfectly fits with Theorem 1.2

\section{REFERENCES}

[1] L. Andersson, P. Chruściel, H. Friedrich, On the regularity of solutions to the Yamabe equation and the existence of smooth hyperboloidal initial data for Einstein's field equations. Comm. Math. Phys. 149 (1992), no. 3, 587-612

[2] P. Aviles, R. McOwen, Complete conformal metrics with negative scalar curvature in compact Riemannian manifolds. Duke Math. J. 56 (1988), no. 2, 395-398

[3] S. Brendle, Curvature flows on surfaces with boundary, Math. Ann. 324 (2002), 491-519.

[4] D. Burghelea, L. Friedlander, T. Kappeler, Mayer-Vietoris formula for determinants of elliptic operators, J. Funct. Anal. 107 (1992), 34-65.

[5] P. Cherrier, Problèmes de Neumann non linéaires sur les variétés riemanniennes, J. Funct. Anal. 57 (1984), no. 2, 154-206.

[6] E. D'Hoker, D.H. Phong, On determinants of Laplacians on Riemann surfaces Comm. Math. Phys. 104 (1986), 537-545.

[7] J. Edward, S. Wu, Determinant of the Neumann operator on smooth Jordan curves, Proc. AMS 111 (1991), no $2,357-363$.

[8] C. Fefferman, C.R. Graham, Q curvature and Poincaré metric, Math Research Letters 9 (2002), no. 2-3, $139-151$.

[9] D. Fried, Zeta functions of Ruelle and Selberg, Ann. É.N.S. 19 (1986), no 4, 491-517.

[10] D. Fried Analytic torsion and closed geodesics on hyperbolic manifolds. Invent. Math. 84 (1986), no. 3, 523-540

[11] I. Gohberg, E. Sigal, An Operator Generalization of the logarithmic residue theorem and the theorem of Rouché, Math. U.S.S.R. Sbornik, 13 (1970), 603-625.

[12] C.R. Graham, Volume and area renormalizations for conformally compact Einstein metrics, Rend. Circ. Mat. Palermo, Ser.II, Suppl. 63 (2000), 31-42.

[13] C.R. Graham, R. Jenne, L.J. Manson, G.A.J. Sparling, Conformally invariant powers of the Laplacian. I. Existence, J. London Math. Soc. (2) 46 (1992), 557-565.

[14] C.R. Graham, J.M. Lee, Einstein metrics with prescribed conformal infinity on the ball, Adv. Math. 87 (1991), no. 2, 186-225.

[15] C.R. Graham, M. Zworski, Scattering matrix in conformal geometry, Invent. Math. 152 (2003), 89-118. 
[16] C. Guillarmou, Meromorphic properties of the resolvent for asymptotically hyperbolic manifolds, Duke Math. J. 129 no 1 (2005), 1-37.

[17] C. Guillarmou, Generalized Krein formula, determinant and Selberg zeta functions in even dimension, Arxiv math.SP/0512173

[18] C. Guillarmou, Resonances and scattering poles on asymptotically hyperbolic manifolds, Math. Research Letters 12 (2005), 103-119.

[19] L. Guillopé, Sur la distribution des longueurs des géodésiques fermées d'une surface compacte à bord totalement géodésique, Duke Math. J. 53 (1986), no. 3, 827-848.

[20] L. Guillopé, M. Zworski Upper bounds on the number of resonances for non-compact complete Riemann surfaces, J. Funct. Anal. 129 (1995), 364-389.

[21] L. Guillopé, M. Zworski, Scattering asymptotics for Riemann surfaces, Ann. Math. 145 (1997), 597-660.

[22] M. Joshi, A. Sá Barreto, Inverse scattering on asymptotically hyperbolic manifolds, Acta Math. 184 (2000), 41-86.

[23] T. Kato, perturbation theory for linear operators, Reprint of the 1980 edition. Classics in Mathematics. Springer-Verlag, Berlin, 1995.

[24] M. Kontsevich, S. Vishik, Determinants of elliptic pseudo-differential operators, Arxiv hep-th/9404046

[25] R. Mazzeo, Regularity for the singular Yamabe problem. Indiana Univ. Math. J. 40 (1991), no. 4, 1277-1299.

[26] R. Mazzeo, R. Taylor, Curvature and uniformization, Israel J. Math. 130 (2002), 323-346.

[27] A. McIntyre, L. A. Takhtajan, Holomorphic factorization of determinants of Laplacians on Riemann surfaces and a higher genus generalization of Kronecker's first limit formula, Geom. Funct. Anal. 16 (2006), 12911323.

[28] K. Okikiolu, Critical metrics for the determinant of the Laplacian in odd dimension, Annals of Mathematics 153 (2001), 471-531.

[29] B. Osgood, R. Phillips, P. Sarnak, Compact isospectral sets of surfaces, J. Funct. Anal. 80 (1988), 212-234.

[30] S. Patterson, The Selberg zeta function of a Kleinian group, in Number Theory, Trace formula and discrete groups: Symposium in honour of Atle Selberg, Oslo, Norway July 14-21, 1987, New-York, Academic Press, 1989.

[31] S. Patterson, P. Perry, The divisor of Selberg's zeta function for Kleinian groups. Appendix A by Charles Epstein., Duke Math. J. 106 (2001), 321-391.

[32] S. Paycha, S. Scott, An explicit Laurent expansion for regularized integrals of holomorphic symbols, preprint Arxiv.

[33] D.B. Ray, I.M. Singer, R-Torsion and the Laplacian on Riemannian manifolds Advances in Math. 7 (1971), 145-210.

[34] P. Sarnak, Determinants of Laplacian, Comm. Math. Phys. 110 (1987), 113-120.

[35] R.T. Seeley Complex powers of an elliptic operator, in 1967 Singular Integrals (Proc. Sympos. Pure Math., Chicago, Ill., 1966), 288-307. Amer. Math. Soc., Providence, R.I.

[36] M. E. Taylor, Partial differential equations, II, Applied Mathematical Sciences 116, Springer, 1996.

[37] A. Voros, Spectral functions, special functions and the Selberg zeta function, Comm. Math. Phys. 110 (1987), 439-465.

Laboratoire J. Dieudonné, CNRs, Université de Nice, Parc Valrose, 06100 Nice, France E-mail address: cguillar@math.unice.fr

Laboratoire J. Leray, CNRS, Université de Nantes, 2, rue de la Houssinière, BP 92208, 44322 NANTES CEDEX 03, France

E-mail address: laurent.guillope@univ-nantes.fr 